\title{
Energy-Efficient Resource Allocation for Multiband UWB Communication Systems
}

\author{
W. Pam Siriwongpairat, Zhu Han, and K. J. Ray Liu, \\ Department of Electrical and Computer Engineering and Institute for Systems Research, \\ University of Maryland, College Park, MD 20742.
}

\begin{abstract}
The emerging ultra-wideband (UWB) system offers a great potential for the design of high-speed short-range communications. However, UWB faces a significant challenge in achieving a low transmit power level, while assuring an adequate system performance. An efficient management of the limited power is thus a key feature to fully exploit the advantages of UWB. In this paper, a cross layer multiuser multiband UWB scheme is proposed to obtain the optimal subband and power allocation strategy. Optimization criteria involve minimization of power consumption under the constraints on the packet error rate, transmission rate, and FCC regulations. To reduce the problem complexity, which is found to be $N P$ hard, a computationally inexpensive suboptimal approach is developed. Simulation results under UWB channel model specified in the IEEE 802.15.3a standard show that the proposed algorithm achieves comparable performances to those of the complex optimal full search approach, and it can save up to $61 \%$ of transmit power compared to the multiband scheme in the standard proposal.
\end{abstract}

\section{INTRODUCTION}

Ultra-wideband (UWB) is an emerging technology that offers great promises to satisfy the growing demand for low cost and high-speed digital wireless home networks. A traditional UWB technology is based on single-band systems [1], [2] that directly modulate data into a sequence of pulses which occupy the available bandwidth of $7.5 \mathrm{GHz}$. Recently, multiband UWB schemes were proposed in [3]-[5], in which the UWB frequency band is divided into several subbands, each with a bandwidth of at least $500 \mathrm{MHz}$ in compliance with the federal communications commission (FCC) regulations [6]. By interleaving the transmitted symbols across subbands, multiband UWB systems can still maintain the average transmit power as if the large $\mathrm{GHz}$ bandwidth is used. The advantage is that the information can be processed over much smaller bandwidth, thereby reducing overall design complexity, as well as improving spectral flexibility and worldwide compliance.

The current leading proposal for the IEEE 802.15.3a wireless personal area networking (WPAN) standard [7] is based on multiband orthogonal frequency division multiplexing (OFDM), which utilizes a combination of OFDM and time-frequency interleaving [5]. The OFDM technique is efficient at collecting multipath energy in highly dispersive channels, as is the case for most UWB channels. Time-frequency interleaving allows the OFDM symbols to be transmitted on different subbands. By using proper timefrequency codes, multiband UWB system provides both frequency diversity and multiple access capability [5].

To this date, most research efforts on multiband UWB systems have been devoted to the physical layer issues [5], [8]. Nevertheless, research and development related to cross layer design is still limited. Some of the key issues that remain largely unexplored are power control and channel allocation. The current multiband proposal divides the subbands into groups, each comprising two to three subbands. A set of certain time-frequency codes is used to interleave the data within each band group [5]. This strategy lacks of the ability to allocate subbands and power optimally since the available subbands are not assigned to each user according to his/her channel condition, and each user's transmit power is equally distributed among subbands without any power adaptation.

Since many applications enabled by UWB are expected to be in portable devices, low power consumption becomes an essential requirement. The low transmit power of UWB emissions not only ensures long life-time for the energy-limited devices, but also reduces co-channel interference. This motivates us to design a proper cross layer algorithm that allows UWB systems to operate at a low transmit power level, while still achieving desired performance.

In this paper, we propose a novel cross layer channel allocation scheme for multiband multiuser UWB wireless systems. The proposed scheme is able to efficiently allocate the subbands as well as transmit power among all users, and hence greatly reduce power consumption without compromising performances. We formulate subband assignment and power allocation problem as an optimization problem whose goal is to minimize the overall transmit power provided that all users achieve their requested transmission rates and desired packet error rate (PER), while the power spectral density complies with the FCC regulations [6]. To reduce the complexity of the problem, which is found to be $N P$ hard, we propose a fast suboptimal algorithm that can guarantee to obtain a near optimal solution, but requires low computational complexity. Simulation results based on UWB channel model specified in the IEEE 802.15.3a standard [9] show that the proposed algorithm achieves up to $61 \%$ of transmit power saving compared to standard multiband scheme [5].

The rest of the paper is organized as follows. Section II describes the system model of multiband UWB. In Section III, we first formulate the power controlled channel allocation problem. Then, a fast suboptimal scheme is developed. Simulation results are given in Section IV and conclusions are drawn in Section V.

\section{System Model}

We consider a UWB system using multiband OFDM that has been proposed for the IEEE 802.15.3a WPAN standard [7]. As shown in Fig. 1, the available UWB spectrum, from $3.1 \mathrm{GHz}$ to $10.6 \mathrm{GHz}$, is divided into $S$ subbands. Each subband occupies a bandwidth of at least $500 \mathrm{MHz}$ in compliance with the FCC regulations. The UWB system employs OFDM with $N$ subcarriers, which are modulated using quadrature phase shift keying 


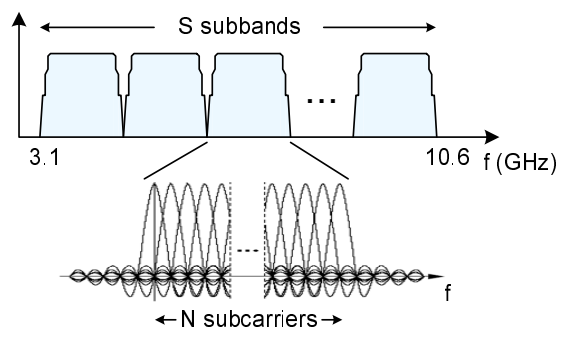

Fig. 1: Spectrum of a Mulitband OFDM System.

TABLE I: Rate-Dependent Parameters

\begin{tabular}{|c|c|c|c|c|}
\hline $\begin{array}{c}\text { Data } \\
\text { Rate } \\
\text { (Mbps) }\end{array}$ & $\begin{array}{c}\text { Modu- } \\
\text { lation }\end{array}$ & $\begin{array}{c}\text { Coding } \\
\text { rate }\end{array}$ & $\begin{array}{c}\text { Conjugate } \\
\text { Symmetric } \\
\text { Inputs to IFFT }\end{array}$ & $\begin{array}{c}\text { Time } \\
\text { Spreading } \\
\text { Factor }\end{array}$ \\
\hline 53.3 & QPSK & $1 / 3$ & Yes & 2 \\
55 & QPSK & $11 / 32$ & Yes & 2 \\
80 & QPSK & $1 / 2$ & Yes & 2 \\
106.7 & QPSK & $1 / 3$ & No & 2 \\
110 & QPSK & $11 / 32$ & No & 2 \\
160 & QPSK & $1 / 2$ & No & 2 \\
200 & QPSK & $5 / 8$ & No & 2 \\
320 & QPSK & $1 / 2$ & No & 1 \\
400 & QPSK & $5 / 8$ & No & 1 \\
480 & QPSK & $3 / 4$ & No & 1 \\
\hline
\end{tabular}

(QPSK). At each OFDM symbol period, the modulated symbol is transmitted over one of the $S$ subbands. These symbols are time-interleaved across subbands. Different bit rates are achieved by using different channel coding, frequency spreading, or time spreading rates. The frequency domain spreading is obtained by choosing conjugate symmetric inputs to the IFFT, while the time-domain spreading is achieved by repeating the same information in an OFDM symbol on two different subbands [5]. The receiver combines the information transmitted via different times or frequencies to increase the signal-to-noise ratio (SNR) of received data.

As listed in Table I, the multiband UWB system provides data rates ranging from 53.3 Mbps to $480 \mathrm{Mbps}$. For the rates not higher than $80 \mathrm{Mbps}$, both time and frequency spreadings are performed, yielding the overall spreading gain of four. In case of rates between 106.7 and $200 \mathrm{Mbps}$, only time-domain spreading is utilized which results in the overall spreading gain of two. The system with information rates higher than $200 \mathrm{Mbps}$ exploits neither frequency nor time spreading, and its the overall spreading gain is one. Forward error correction codes with coding rates of $1 / 3,11 / 32,1 / 2,5 / 8$ or $3 / 4$ are employed to provide different channel protections with various information data rates.

In what follows, we will describe the channel model and system model for the multiuser multiband UWB system.

\section{A. Channel Model}

The channel model specified in the IEEE 802.15.3a standard [9] is a modified version of the Saleh-Valenzuela (SV) model for indoor channels [10]. The channel impulse response for the $k^{t h}$ user can be represented by

$$
h_{k}(t)=X_{k} \sum_{m=0}^{M_{k}} \sum_{l=0}^{L_{k}} \alpha_{k}(m, l) \delta\left(t-T_{k}(m)-\tau_{k}(m, l)\right),
$$

where $X_{k}$ is the log-normal shadowing, and $\alpha_{k}(m, l)$ denotes the gain of the $l^{\text {th }}$ multipath component in the $m^{\text {th }}$ cluster. The

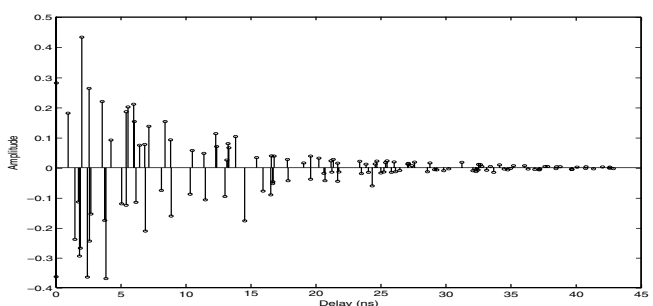

(a) Impulse Response

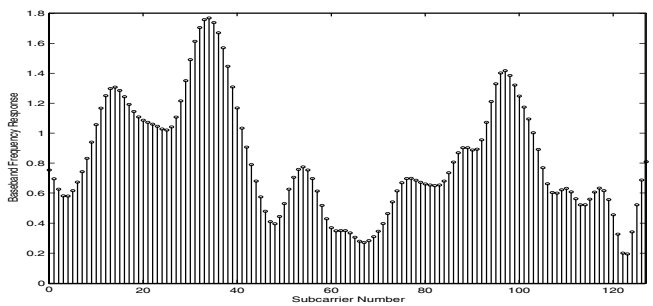

(b) Baseband Frequency Response

Fig. 2: A Realization of UWB Standard Channel Model 1.

time duration $T_{k}(m)$ represents the delay of the $m^{\text {th }}$ cluster, and $\tau_{k}(m, l)$ is the delay of the $l^{t h}$ path in the $m^{\text {th }}$ cluster relative to the cluster arrival time. The cluster arrivals and the path arrivals within each cluster can be modeled as Poisson distribution with rate $\Lambda_{k}$ and rate $\lambda_{k}\left(\lambda_{k}>\Lambda_{k}\right)$, respectively. The path amplitude $\left|\alpha_{k}(m, l)\right|$ follows the log-normal distribution, whereas the phase $\angle \alpha_{k}(m, l)$ is a uniform random variable over $[0,2 \pi)$. The model parameters corresponding to several scenarios are provided in [9]. With the choice of cyclic prefix length greater than the duration of the channel impulse response, OFDM allows for each UWB subband to be divided into a set of $N$ orthogonal narrowband channels. The baseband frequency response at the $n^{\text {th }}(n=0,1, \ldots, N-1)$ subcarrier is given by

$$
H_{k}(n)=X_{k} \sum_{m=0}^{M_{k}} \sum_{l=0}^{L_{k}} \alpha_{k}(m, l) e^{-\mathrm{j} 2 \pi n \Delta f\left(T_{k}(m)+\tau_{k}(m, l)\right)},
$$

where $\mathrm{j}=\sqrt{-1}$, and $\Delta f$ is the frequency separation between two adjacent subcarriers. Fig. 2 illustrates a channel time-domain realization and its corresponding baseband frequency response, using parameters for channel model 1 [9].

\section{B. System Model: Subband Assignment and Power Allocation}

We consider a multiuser multiband UWB scenario where $K$ users simultaneously transmit their information. The $k^{t h}$ user has the transmission rate $R_{k}$, which can be any value specified in Table I. As shown in Table I, if the rate is higher than $200 \mathrm{Mbps}$, there is no time spreading; otherwise, the time-domain spreading operation is performed with a spreading factor of two. In this case, any time-frequency code with a period of two can guarantee that each user will achieve the additional diversity by transmitting the same information over two OFDM blocks. The time-frequency codes with period longer than two can also be used to improve the multiple access capability for asynchronous UWB wireless networks [5]. To simplify the problem formulation, we consider in this paper a multiband UWB system employing time-frequency codes of length two. The extension to UWB systems with longer time-frequency codes is straight forward. 
To specify in which frequency-bands each user can transmit his/her information, we define a $K \times S$ assignment matrix A, whose $(k, s)^{t h}$ element is denoted by $a_{k s}$, for $k=1,2, \ldots, K$ and $s=1,2, \ldots, S$. This $a_{k s}$ represents the number of OFDM symbols that user $k$ is allowed to transmit on the $s^{t h}$ subband during two OFDM symbol periods. Assuming that each user utilizes one subband per transmission, $a_{k s}$ can take any value from the set $\{0,1,2\}$. However, when the $k^{\text {th }}$ user's transmission rate is less than or equal to $200 \mathrm{Mbps}$, we need to ensure that the band hopping is performed to obtain the diversity from time spreading. In this case, $a_{k s}$ is restricted to $a_{k s} \in\{0,1\}$. Thus, the element of assignment matrix satisfies

$$
a_{k s} \in \phi\left(R_{k}\right)= \begin{cases}\{0,1\}, & R_{k} \leq 200 \mathrm{Mbps} ; \\ \{0,1,2\}, & R_{k}>200 \mathrm{Mbps} .\end{cases}
$$

During each OFDM symbol period, one user will occupy one subband. Since we consider the duration of two OFDM blocks, the assignment strategy needs to satisfy

$$
\sum_{s=1}^{S} a_{k s}=2, \quad k=1,2, \ldots, K .
$$

In addition, to minimize the multiple access interference, each subband is assigned to a specific user at a time, and hence each subband can be used at most twice during two OFDM symbol periods. Therefore, the subband assignment also follows

$$
\sum_{k=1}^{K} a_{k s} \leq 2, \quad s=1,2, \ldots, S .
$$

Let $P_{k}^{s}(n)$ denote the $k^{t h}$ user's transmit power at subcarrier $n$ of the $s^{t h}$ subband. Accordingly, the SNR of user $k$ at the $s^{t h}$ subband and the $n^{\text {th }}$ subcarrier is given by

$$
\Gamma_{k}^{s}(n)=\frac{P_{k}^{s}(n) G_{k}^{s}(n)}{\sigma_{k}^{2}},
$$

where $G_{k}^{s}(n)$ is the corresponding channel gain. We can express $G_{k}^{s}(n)$ as

$$
G_{k}^{s}(n)=\left|H_{k}^{s}(n)\right|^{2}\left(\frac{4 \pi d_{k}}{\lambda_{k}^{s}}\right)^{-\nu}
$$

in which $H_{k}^{s}(n)$ is the channel frequency response at subband $s$ and subcarrier $n, \nu$ is the propagation loss factor, $d_{k}$ represents the distance between the transmitter and receiver, $\lambda_{k}^{s}=3 \times 10^{8} / f_{c, k}^{s}$ is the wavelength of the transmitted signal, and $f_{c, k}^{s}$ is the center frequency of the waveform. In (6), $\sigma_{k}^{2}$ denotes the noise power at each subcarrier, which is defined as

$$
\sigma_{k}^{2}=2 \times 10^{\left(-174+10 \log _{10}\left(R_{k}\right)+N_{F}\right) / 10},
$$

where $R_{k}$ is the $k^{t h}$ user's data rate, and $N_{F}$ is the received noise figure referred to the antenna terminal [5]. As in multiband standard, the noise power is assumed the same for every subcarrier.

Due to the consideration for the simple transceiver of UWB, the current standard assumes that there is no bit loading and the power is equally distributed across subcarriers within each subband. Similarly, we assume that $P_{k}^{s}(n)=P_{k}^{s}\left(n^{\prime}\right)$ for any $0 \leq n, n^{\prime} \leq N-1$. Denote

$$
P_{k}^{s}(n)=P_{k}^{s}, \quad n=0,1, \ldots, N-1,
$$

then the $K \times S$ power allocation matrix can be defined as $[\mathbf{P}]_{k s}=P_{k}^{s}$, in which $(k, s)^{t h}$ component represents the $k^{t h}$ user's transmit power in subband $s$.

\section{Multiband Resource Allocation Algorithm}

In multiband frequency band plan [5], the subband center frequencies span a wide range from $3.43 \mathrm{GHz}$ to $10.3 \mathrm{GHz}$. Consequently, different subbands tend to undergo different fading and propagation loss. Additionally, the channel condition for a specific subband may be good for more than one user. Therefore, to efficiently reduce the power consumption, we need to optimize subband assignment matrix $\mathbf{A}$ and power allocation matrix $\mathbf{P}$ under some practical constraints.

In this section, first, we derive a generalized SNR expression for various UWB transmission modes. Second, we provide a necessary condition for the SNR so as to satisfy the PER requirement. Then, we propose a problem formulation to minimize the overall transmit power provided that all users achieve their requested transmission rates and desired PER, while the transmit power level is below the FCC limitation and rate parameters are according to the standard proposal given in Table I. Finally, we develop a fast suboptimal scheme to solve the proposed problem.

\section{A. Generalized SNR for Different Transmission Modes}

Assume that the channel state information is perfectly known at the receiver. The receiver employs a maximum ratio combiner (MRC) to combine the information transmitted via different times or frequencies. As a result, the average SNR at the output of MRC depends not only on the channel coding rate, but also the time and frequency spreading factors. The following proposition provides a generalized expression of the average SNR for any data rates.

Proposition 1 Assume maximum ratio combining and $P_{k}^{s}(n)=$ $P_{k}^{s}$ for all subcarrier $n$, then the $k^{\text {th }}$ user's average SNR is given by

$$
\bar{\Gamma}_{k}=\sum_{s=1}^{S} a_{k s} P_{k}^{s} F_{k}^{s}
$$

where

$$
F_{k}^{s} \triangleq \frac{b_{k}}{N \sigma_{k}^{2}} \sum_{n=0}^{N-1} G_{k}^{s}(n)
$$

and $b_{k}$ is a constant that depends on the $k^{\text {th }}$ user's information data rate as follows:

$$
b_{k}= \begin{cases}2, & R_{k} \leq 80 \text { Mbps } \\ 1, & 80<R_{k} \leq 200 \mathrm{Mbps} ; \\ 1 / 2, & R_{k}>200 \mathrm{Mbps} .\end{cases}
$$

Proof: Recall that when $R_{k}$ is not higher than $80 \mathrm{Mbps}$, the information is spread across both time and frequency with the overall spreading gain of four. Consequently, the total SNR for the $k^{t h}$ user at subcarrier $n, n=0,1, \ldots, N / 2-1$, is

$$
\Gamma_{k}(n)=\sum_{s=1}^{S} a_{k s}\left[\Gamma_{k}^{s}(n)+\Gamma_{k}^{s}(n+N / 2)\right] .
$$

Average (13) over $N / 2$ subcarriers, resulting in the average SNR

$$
\bar{\Gamma}_{k}=\frac{1}{N / 2} \sum_{n=0}^{N / 2-1} \Gamma_{k}(n)=\frac{1}{N / 2} \sum_{n=0}^{N-1} \sum_{s=1}^{S} a_{k s} \Gamma_{k}^{s}(n) .
$$

By substituting (6) into (14) and assuming $P_{k}^{s}(n)=P_{k}^{s}$, we obtain 


$$
\bar{\Gamma}_{k}=\sum_{s=1}^{S} a_{k s} P_{k}^{s}\left(\frac{2}{N \sigma_{k}^{2}} \sum_{n=0}^{N-1} G_{k}^{s}(n)\right) .
$$

When $R_{k}$ is between 106.7 and $200 \mathrm{Mbps}$, only time spreading is performed, and hence the total SNR at subcarrier $n, n=$ $0,1, \ldots, N-1$, becomes

$$
\Gamma_{k}(n)=\sum_{s=1}^{S} a_{k s} \Gamma_{k}^{s}(n)=\sum_{s=1}^{S} a_{k s} \frac{P_{k}^{s}(n) G_{k}^{s}(n)}{\sigma_{k}^{2}} .
$$

Thus, the average SNR can be obtained from (16) as

$$
\bar{\Gamma}_{k}=\frac{1}{N} \sum_{n=0}^{N-1} \Gamma_{k}(n)=\sum_{s=1}^{S} a_{k s} P_{k}^{s}\left(\frac{1}{N \sigma_{k}^{2}} \sum_{n=0}^{N-1} G_{k}^{s}(n)\right) .
$$

For $R_{k}$ higher than $200 \mathrm{Mbps}$, there is no spreading and the $k^{t h}$ user's average SNR is simply the average of $\Gamma_{k}^{s}(n)$ over $N$ subcarriers and two subbands, i.e.,

$$
\begin{aligned}
\bar{\Gamma}_{k} & =\frac{1}{2 N} \sum_{n=0}^{N-1} \sum_{s=1}^{S} a_{k s} \Gamma_{k}^{s}(n) \\
& =\sum_{s=1}^{S} a_{k s} P_{k}^{s}\left(\frac{1}{2 N \sigma_{k}^{2}} \sum_{n=0}^{N-1} G_{k}^{s}(n)\right) .
\end{aligned}
$$

Express (15), (17) and (18) in terms of $F_{k}^{s}$ defined in (11) leading to the results in (10).

\section{B. PER and Rate Constraint}

A common performance requirement of UWB systems is to offer packet transmission with an error probability less than a desired threshold value. The PER metric is directly related to the bit error rate (BER) performance, which in turn depends on the SNR at the output of the MRC. By keeping the SNR level higher than a specific value, the PER can be ensured to be lower than the PER threshold.

Suppose the maximum PER is $\varepsilon$ and the packet length is $L$ bits, then the bit error probability after the channel decoder for the $k^{t h}$ user, $\mathcal{P}_{k}$, needs to satisfy

$$
1-\left(1-\mathcal{P}_{k}\right)^{L} \leq \varepsilon
$$

By the assumptions of the use of convolutional coding and Viterbi decoding with perfect interleaving, $\mathcal{P}_{k}$ is given by [11]

$$
\mathcal{P}_{k} \leq \sum_{d=d_{\text {free }}}^{\infty} a_{d} \mathcal{P}_{k}(d)
$$

where $d_{\text {free }}$ is the free distance of the convolutional code, $a_{d}$ denotes the total number of error events of weight $d$, and $\mathcal{P}_{k}(d)$ represents the probability of choosing the incorrect path with distance $d$ from the correct path. Assume hard-decision decoding, then $\mathcal{P}_{k}(d)$ is related to the average BER, $\bar{B}_{k}$, as [11]

$$
\mathcal{P}_{k}(d)=\sum_{l=(d+1) / 2}^{d}\left(\begin{array}{c}
d \\
l
\end{array}\right) \bar{B}_{k}^{l}\left(1-\bar{B}_{k}\right)^{d-l}
$$

when $d$ is odd, and

$$
\mathcal{P}_{k}(d)=\sum_{l=\frac{d}{2}+1}^{d}\left(\begin{array}{c}
d \\
l
\end{array}\right) \bar{B}_{k}^{l}\left(1-\bar{B}_{k}\right)^{d-l}+\frac{1}{2}\left(\begin{array}{c}
d \\
\frac{d}{2}
\end{array}\right) \bar{B}_{k}^{\frac{d}{2}}\left(1-\bar{B}_{k}\right)^{\frac{d}{2}}
$$

when $d$ is even. The average BER $\bar{B}_{k}$ can be obtained by averaging the conditional BER over the probability density function of the SNR at the output of MRC. With $\Gamma_{k}$ denoting the instantaneous SNR at the MRC output, the conditional BER is given by [11]

$$
B_{k}\left(\Gamma_{k}\right)=\mathrm{Q}\left(\sqrt{\Gamma_{k}}\right) \text {, }
$$

where $\mathrm{Q}(\cdot)$ is the Gaussian error function.

From (19) and (20), we can see that for a given value of PER threshold $\varepsilon$, a corresponding BER threshold can be obtained. Since the error probability $\mathcal{P}_{k}$ in (20) is related to the coding rate through the parameters $d_{f r e e}$ and $a_{d}$, the BER requirement depends not only on the value of $\varepsilon$, but also on the data rate $R_{k}$. This implies that the SNR threshold is also a function of both $\varepsilon$ and $R_{k}$. Let $\gamma\left(\varepsilon, R_{k}\right)$ be the $k^{t h}$ user's minimum SNR that is required to achieve the data rate $R_{k}$ with PER less than $\varepsilon$. Then, the necessary condition for the average SNR (defined in (10)) to satisfy the PER requirement is given by

$$
\bar{\Gamma}_{k}=\sum_{s=1}^{S} a_{k s} P_{k}^{s} F_{k}^{s} \geq \gamma\left(\varepsilon, R_{k}\right)
$$

\section{Problem Formulation}

The optimization goal is to minimize the overall transmit power subject to the PER, rate, and FCC regulation constraints. Recall from (3) that the assignment matrix $\mathbf{A}$ has $a_{k s} \in \phi\left(R_{k}\right), \forall k, s$. We can formulate the problem as follows:

$$
\min _{\mathbf{A}, \mathbf{P}} P_{\text {sum }}=\sum_{k=1}^{K} \sum_{s=1}^{S} a_{k s} P_{k}^{s}
$$

$$
\text { s.t. }\left\{\begin{array}{l}
\text { Rate and PER: } \sum_{s=1}^{S} a_{k s} P_{k}^{s} F_{k}^{s} \geq \gamma\left(\varepsilon, R_{k}\right), \forall k ; \\
\text { Assignment (4): } \sum_{s=1} a_{k s}=2, \forall k ; \\
\text { Assignment (5): } \sum_{k=1}^{K} a_{k s} \leq 2, \forall s ; \\
\text { Power: } P_{k}^{s} \leq \bar{P}, \forall k, s,
\end{array}\right.
$$

where the first constraint in (23) is to ensure rate and PER requirements. The second and third constraints are described in Section II-B. The last constraint is related to the limitation on transmit power spectral density of $-41.3 \mathrm{dBm} / \mathrm{MHz}$, according to FCC Part 15 rules [6]. Here, $\bar{P}$ is the maximum power after taking into consideration the effects such as peak-to-average ratio.

If the elements in the assignment matrix $\mathbf{A}$ are binary, the problem defined in (23) can be viewed as a generalized form of generalized assignment problem [12] which is $N P$ hard. Since the components of $\mathbf{A}$ can be 0,1 , or 2, the problem is an even harder integer programming problem. So the existing channel assignment solutions are not applicable in (23). Although the optimal solution can be found through full search, it is computationally expensive. To overcome the complexity issue, we propose in the subsequent subsection a fast suboptimal scheme, which is near optimal but has very low computational complexity.

\section{Subband Assignment and Power Allocation Algorithm}

The basic idea is a greedy approach to assign $a_{k s}$ for a user step by step, so that the power consumption is minimized. The initialization is to set $\mathbf{A}=\mathbf{0}_{K \times S}$, define the user optimization list $K_{\text {live }}=\{1,2, \ldots, K\}$, and define the subband optimization 
TABLE II: Fast Suboptimal Algorithm

\begin{tabular}{|l|}
\hline Initialization: \\
$\quad a_{k s}=0, \forall k, s, K_{\text {live }}=\{1, \ldots, K\}, S_{\text {live }}=\{1, \ldots, S\}$ \\
\hline Repeat Until $K_{\text {live }}=\emptyset$ or $S_{\text {live }}=\emptyset$ \\
For $k \in K_{\text {live }}$ \\
$\quad P_{d u m m y}^{k}=\min \sum_{s=1}^{S} w_{s} a_{k s} P_{k}^{s}$ s.t. $a_{k s} \in S_{\text {live }}$ \\
End \\
Select $k^{\prime}$ with the maximal $P_{d u m m y}^{k}, \forall k$, assign the \\
$\quad$ corresponding $a_{k^{\prime} s}$ to $\mathbf{A}$, and update $\mathbf{P}$. \\
$K_{\text {live }}=K_{\text {live }} \backslash k^{\prime}$. \\
If $\sum_{k=1}^{K} a_{k s^{\prime}}=2, S_{\text {live }}=S_{\text {live }} \backslash s^{\prime}, \forall s^{\prime}$. \\
\hline If $(\max (\mathbf{P})>P)$ or $\left(S_{\text {live }}=\emptyset\right.$ and $\left.K_{\text {live }} \neq \emptyset\right)$, an outage is reported; \\
else Return $\mathbf{A}$ and $\mathbf{P}$.
\end{tabular}

list $S_{\text {live }}=\{1,2, \ldots, S\}$. First, each user makes a hypothesis that he/she can assign his/her transmission into different subbands regarding absence of other users. For each hypothesis, a dummy overall transmission power $P_{d u m m y}^{k}$ is calculated. The user with the highest dummy overall transmit power to achieve his/her rate will be assigned first, so that the best channel is assigned to the user that can reduce the overall power most. Then, this user is removed from the optimization list $K_{\text {live }}$. Since each subband can only accommodate one user per symbol period and we consider two OFDM symbol periods, when a subband is assigned twice, this subband is removed from the optimization list $S_{\text {live }}$. Then, we go to the first step for the rest of the users to assign their transmissions into the rest of the subbands. This iteration is continued until all users are assigned with their subbands, i.e., $K_{\text {live }}=\emptyset$. Finally, the algorithm checks if the maximum power is larger than the power limitation. If yes, an outage is reported; otherwise, the final values of $\mathbf{A}$ and $\mathbf{P}$ are obtained. The proposed algorithm is described in Table II.

The complexity of the proposed algorithm is only $O\left(K^{2} S\right)$. Although the algorithm is suboptimal, simulation results illustrated in the succeeding section shows that the proposed fast suboptimal algorithm has very close performances to the optimal solutions obtained by full search. Another complexity issue is that for the proposed scheme, power control is needed for each subband. This will increase the system complexity slightly, but from the simulation results, we can see that the performance improvement is significant. Moreover, the proposed algorithm can be implemented by the master node to manage the power and subband usages of all users in a UWB picocell system, as adopted in the IEEE 802.15.3a standard [7].

\section{Simulation Results}

To illustrate the performance of the proposed schemes, we perform simulations for multiband UWB systems with $N=128$ subcarriers, $S=14$ subbands, and the subband bandwidth of 528 MHz. Following the IEEE 802.15.3a standard proposal [5], we utilize the subbands with center frequencies $2904+528 \times n_{b}$ $\mathrm{MHz}, n_{b}=1,2, \ldots, 14$. The OFDM symbol is of duration $T_{F F T}=242.42 \mathrm{~ns}$. After adding the cyclic prefix of length $T_{C P}=60.61 \mathrm{~ns}$ and the guard interval of length $T_{G I}=9.47 \mathrm{~ns}$, the symbol duration becomes $T_{S Y M}=312.5 \mathrm{~ns}$. The maximum transmit power is $-41.3 \mathrm{dBm} / \mathrm{MHz}$, and the PER is maintained such that PER $<8 \%$ for a 1024 byte packet. The average noise power follows (8) with $N_{F}=6.6 \mathrm{~dB}$, and the propagation loss factor is $\nu=2$.
We consider a multiuser scenario in which each user is located at a distance of less than 4 meters from the central base station. The performance is evaluated in multipath channel environments specified in the IEEE 802.15.3a channel modeling sub-committee report [9]. We employ channel model 1 and 2, which are based on channel measurements over the range of 0-4 meters.

In what follows, we present the average transmit power and the outage probability curves for multiband UWB systems. Here, the outage probability is the probability that the requested rate cannot be supported under the constraints in (23). We compare the performances of the proposed scheme with those of the current multiband scheme in the standards proposal [5].

For Fig. 3(a) and Fig. 3(b), the number of users is fixed to $K=3$, while each user is randomly located at the distance of 1 to 4 meters from the base station. In Fig. 3(a), we illustrate the average transmit power as a function of the transmission rates for standard multiband scheme, the proposed fast suboptimal scheme, and the optimal scheme obtained by full search. It is apparent that the proposed algorithm greatly reduces the average transmit power compared to that in standard proposal. In addition, the proposed algorithm can achieve almost the same performance to the optimal scheme. The results show that both fast suboptimal and optimal approach can reduce about $60 \%$ of average transmit power at low rates (53.3-200 Mbps) and up to 35\% at high rates (320-480 Mbps). Notice that the curves are not smooth because of the discrete nature of the problem.

Fig. 3(b) shows the outage probability versus the transmission rates. As we can see, all three schemes have non-zero outage probability when the rate is higher than $200 \mathrm{Mbps}$. This is because the system under current channel conditions cannot support such rates within the constraints. In these cases, the proposed scheme achieves lower outage probability than that of the standard multiband scheme for any rates. Significant performance difference can be observed at high rates. For instance, at $320 \mathrm{Mbps}$, the outage probability of the proposed scheme is 0.36 , whereas that of the standard multiband scheme is 0.64 .

We also consider a multiuser system with different number of users, each located at a fixed position of about 4 meters from the base station. Specifically, the distance between the $k^{\text {th }}$ user and the base station is specified as $d_{k}=4-0.1(k-1)$ for $k=1,2, \ldots, K$. In Fig. 4(a) and Fig. 4(b), we show the average transmit power and outage probability as functions of number of users for the transmission rates of 110, 160, and 200 Mbps. In both figures, we use the standard multiband scheme and the proposed scheme. We can observe from Fig. 4(a) that the transmit power increases with the number of users. This is because of the limited available subbands with good channel conditions. When the number of users is large, some users have to occupy the subbands with worse channel conditions. Comparing the proposed algorithm with standard multiband approach, we can see that the proposed scheme achieves lower power for all rate requirements.

Fig. 4(b) shows that the outage probability increases with the number of users, especially when the desired rate is high. This is due to the fact that as the number of users increases, the system is more crowded and may not be feasible to support all these users at all times. Observe that at any rate, the performance of the standard multiband scheme degrades as the number of users increases. On 


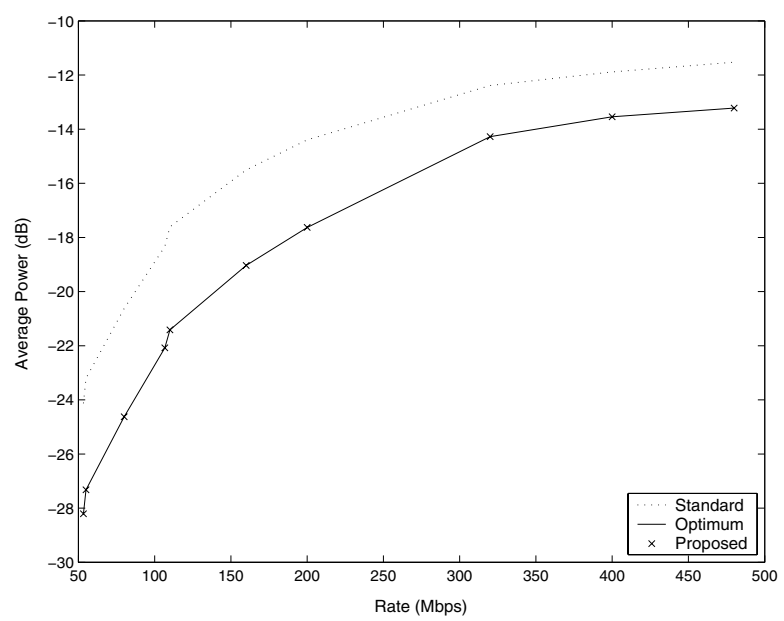

(a) Average Power vs. Rates

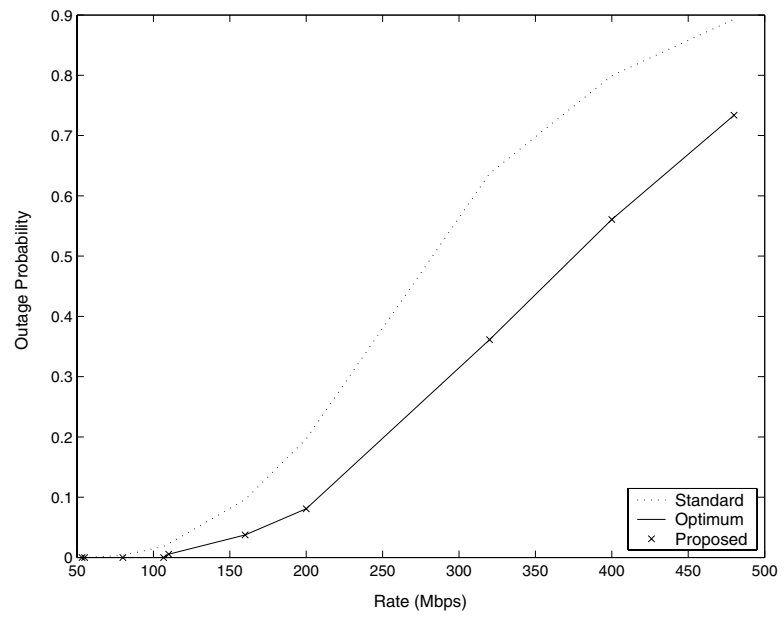

(b) Outage Probability vs. Rates

Fig. 3: Performances of Three-User System with Random Location.

the other hand, when the proposed scheme is employed, the effect of the number of users to the outage probability is insignificant when the rates are not higher than $160 \mathrm{Mbps}$. As we can see, the proposed algorithm achieves smaller outage probabilities than those of the standard scheme under all conditions.

\section{CONCLUSIONS}

Low power consumption is one of the key elements to make multiband UWB technology be the solution for future indoor wireless communications. We propose in this paper an efficient cross layer algorithm for allocating subband and power among users in a multiband UWB system. The proposed scheme aims to reduce power consumption without compromising performance, resulting in much lower co-channel interference and a substantial increase in battery life. We propose a general framework to minimize the overall transmit power under the practical implementation constraints. The formulated problem is NP hard; however, with the proposed fast suboptimal algorithm, we can reduce the computational complexity to only $O\left(K^{2} S\right)$, where $K$ is the number of users and $S$ is the number of subbands. Simulation results show that the proposed algorithm achieves comparable performances to those of complex optimal full search algorithm, and can save up to $61 \%$ of power consumption compared to the standard multiband scheme.

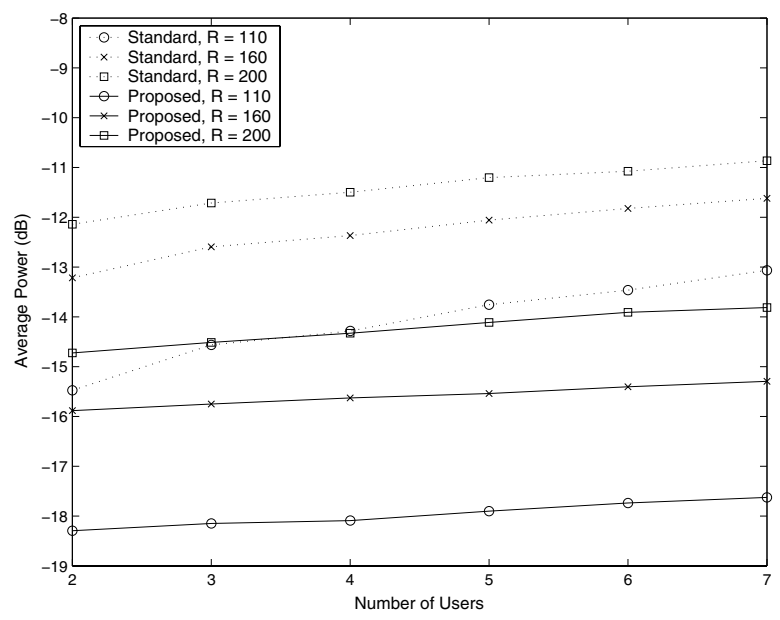

(a) Average Power vs. Number of Users

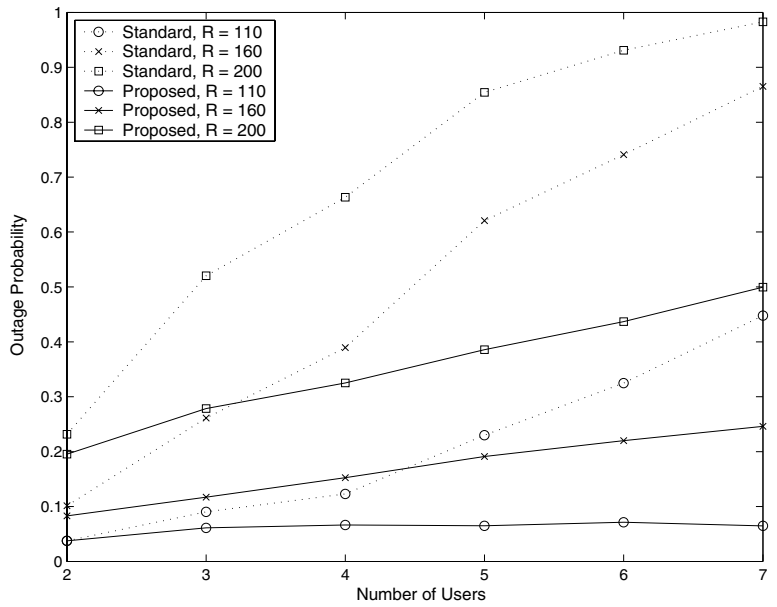

(b) Outage Probability vs. Number of Users

Fig. 4: Performances of Multiple-User System.

\section{REFERENCES}

[1] M. Z. Win and R. A. Scholtz, "Impulse Radio: How It Works," IEEE Commun. Letters, vol. 2, no. 2, pp. 36-38, Feb. 1998.

[2] M. L. Welborn, "System Considerations for Ultra-Wideband Wireless Networks," IEEE Radio and Wireless Conf., pp. 5-8, Aug. 2001.

[3] J. R. Foerster, et. al, "Intel CFP Presentation for a UWB PHY," IEEE P802.15-03/109r1, Mar. 3, 2003.

[4] E. Saberinia and A. H. Tewfik, "Multi-User UWB-OFDM Communications," IEEE Proc. on Pacific Rim Conf. on Commun., Computers and Signal Processing, vol. 1, pp. 127-130, Aug. 2003.

[5] A. Batra, et. al, "Multi-Band OFDM Physical Layer Proposal for IEEE 802.15 Task Group 3a," IEEE P802.15-03/268r3, Mar. 2004.

[6] Federal Communications Commission Report FCC 02-48 "Revision of Part 15 of the Commission's Rules Regarding Ultra-Wideband Transmission Systems, First Report and Order," Apr. 2002.

[7] IEEE 802.15WPAN High Rate Alternative PHY Task Group 3a (TG3a). Internet: www.ieee802.org/15/pub/TG3a.html

[8] Y. Nakache, et. al, "Low-Complexity Ultrawideband Transceiver with Compatibility to Multiband-OFDM," Technical Report, A Mitsubishi Electronic Research Laboratory. Internet: www.merl.com/reports/docs/TR2004051.pdf.

[9] J. R. Foerster, et. al, "Channel Modeling Sub-committee Report Final," IEEE802.15-02/490, Nov. 18, 2003.

[10] A. A. M. Saleh and R. A. Valenzuela, "A Statistical Model for Indoor Multipath Propagation," IEEE J. on Selected Areas in Commun., vol. 5, no. 2, pp. 128-137, Feb. 1987.

[11] J. G. Proakis, Digital Communications, $4^{\text {th }}$ Ed., McGraw-Hill, New York, 2001.

[12] H. Kellerer, U. Pferschy, and D. Pisinger, Knapsack problems, Springer, New York, 2004. 